\title{
AUTHOR INDEX Volume 2
}

Ali, M. S., see Hadi, M. A.

2 (2013) 1350007

Asgari, A. \& Nayebi, A., Implementation of the Eulerian and the Arbitrary Lagrangian-Eulerian Descriptions in Finite Element Simulation of Extrusion Processes

3 \& 4 (2013) 1350013

Balasundar, I., see Satheesh Kumar, S. S.

Barman, S., Das, G. P. \& Kawazoe, Y., First Principles

1 (2013) 1350001

Density Functional Investigation of Supported

Tungsten Cluster $\left(W_{n} ; n=1\right.$ to 6$)$ on Anchored

Graphite (0001) Surface

Bohnke, O., see Gunes, V.

Botquelen, J.-Y., see Gunes, V.

Chamoli, N., see Rattan, M.

Chen, C., see He, T.

Cheng, G.-G., see Zhang, Z.-Q.

Das, G. P., see Barman, S.

Ding, J.-N., see Zhang, Z.-Q.

Do, T. D., Leroy, R. \& Joly, D., Computational Contour of Mixed Mode Crack-Tip Plastic Zone for Aluminum Alloy 2024T351

1 (2013) 1350003

$\mathrm{Gu}, \mathrm{Y}$., see Sauret, E.

3 \& 4 (2013) 1350016

Gu, Y. T., see Zhan, H. F.

3 \& 4 (2013) 1350020

Gunes, V., Botquelen, J.-Y. \& Bohnke, O., Electronic Conductivity Measurements in Solid Electrolytes Using an Ion Blocking Microelectrode: Noise

Rejection Based on a Median Filter

3 \& 4 (2013) 1350015

2 (2013) 1350009

2 (2013) 1350009

1 (2013) 1350005

1 (2013) 1350002

3 \& 4 (2013) 1350017

3 \& 4 (2013) 1350015

3 \& 4 (2013) 1350017

Guo, Z., see Ren, G.

2 (2013) 1350009

2 (2013) 1350010

Guo, Z., see Sun, S.

3 \& 4 (2013) 1350018

Gupta, N., see Rattan, M.

1 (2013) 1350005

Hadi, M. A., Ali, M. S., Naqib, S. H. \& Islam, A. K. M.

A., Band Structure, Hardness, Thermodynamic and Optical Properties of Superconducting $\mathrm{Nb}_{2} \mathrm{AsC}$, 
Hasebe, T., see Kajiwara, N.

Hasebe, T., see Okuda, T.

He, T., Li, Y., Li, H. \& Chen, C., Optimization Design for a Dielectric Elastomer Membrane Actuator

Imiya, K., see Kajiwara, N.

Imiya, K., see Okuda, T.

Inguva, S., see Joglekar, M. M.

Islam, A. K. M. A., see Hadi, M. A.

Joglekar, M. M., Ramakrishnan, N., Inguva, S., Vanimisetti, S. K. \& Moulliet, R. B., Numerical Implementation of Porous Composite

Electrode-Degradation Model to Study the Cycle Life of Li-Ion Cell

Joly, D., see Do, T. D.

Kajiwara, N., Imiya, K. \& Hasebe, T., FTMP-Based Modeling and Simulation of Magnesium

Kang, G., see Luo, J.

Kawazoe, Y., see Barman, S.

Krasnyuk, I. B., Spatial-Temporal Oscillations of the Order Parameter in Confined Diblock Copolymer Mixtures

Lee, A. S. H., see Li, K.

Leroy, R., see Do, T. D.

Li, H., see He, T.

Li, H., see Ren, Y. X.

Li, K., Lee, A. S. H., Zhang, Y.-W. \& Pan, H., Electronic and Magnetic Properties of Silicene and Silicane Nanoribbons

Li, Q., see Ren, G.

Li, Y., see He, T.

Ling, Z.-Y., see Zhang, Z.-Q.

Luo, J., Kang, G. \& Shi, M., Simulation to the Cyclic Deformation of Polycrystalline Aluminum Alloy Using Crystal Plasticity Finite Element Method

Marik, A. K., see Rath, S.

Moulliet, R. B., see Joglekar, M. M.

Naqib, S. H., see Hadi, M. A.

Nayebi, A., see Asgari, A.

Ng, T. Y., see Ren, Y. X.

Okuda, T., Imiya, K. \& Hasebe, T., FTMP-Based

Simulation of Twin Nucleation and Substructure

Evolution Under Hypervelocity Impact
3 \& 4 (2013) 1350022

3 \& 4 (2013) 1350021

1 (2013) 1350002

3 \& 4 (2013) 1350022

3 \& 4 (2013) 1350021

2 (2013) 1350012

2 (2013) 1350007

2 (2013) 1350012

1 (2013) 1350003

3 \& 4 (2013) 1350022

3 \& 4 (2013) 1350019

3 \& 4 (2013) 1350015

1 (2013) 1350006

2 (2013) 1350011

1 (2013) 1350003

1 (2013) 1350002

2 (2013) 1350008

2 (2013) 1350011

2 (2013) 1350010

1 (2013) 1350002

3 \& 4 (2013) 1350017

3 \& 4 (2013) 1350019

1 (2013) 1350004

2 (2013) 1350012

2 (2013) 1350007

3 \& 4 (2013) 1350013

2 (2013) 1350008

3 \& 4 (2013) 1350021 
Pan, H., see Li, K.

Peng, X., see Sun, S.

Raghu, T., see Satheesh Kumar, S. S.

Ramakrishnan, N., see Joglekar, M. M.

Rath, S., Sengupta, P. P., Singh, A. P., Marik, A. K. \& Talukdar, P., Mathematical-Artificial Neural Network Hybrid Model to Predict Roll Force During Hot Rolling of Steel

Rattan, M., Chamoli, N., Singh, S. B. \& Gupta, N., Creep Behavior of Anisotropic Functionally Graded Rotating Discs

Ren, G., Tang, T., Guo, Z., Yang, Y. \& Li, Q., Brittle Fragmentation of an Expanding Ring by Molecular Dynamics

Ren, Y. X., Ng, T. Y. \& Li, H., ssDNA Translocation with Carboxyl-Modified Transmembrane Single-Walled Carbon Nanotube

Saha, S. C., see Sauret, E.

Satheesh Kumar, S. S., Balasundar, I. \& Raghu, T., Finite Element Analysis of Constrained Groove Pressing of Pure Aluminum Sheets

Sauret, E., Saha, S. C. \& Gu, Y., Numerical Simulations of Particle Deposition in Metal Foam Heat Exchangers

Sengupta, P. P., see Rath, S.

Shi, M., see Luo, J.

Singh, A. P., see Rath, S.

Singh, S. B., see Rattan, M.

Sun, S., Peng, X. \& Guo, Z., Simulation of Nonlinear Magnetorheological Particle-Filled Elastomers

Talukdar, P., see Rath, S.

Tang, T., see Ren, G.

Vanimisetti, S. K., see Joglekar, M. M.

Vinogradov, O., Mechanical Properties and Topological Changes in a Fe-Crystal with Fixed and Migrating H-Atom: A Numerical Study

Xia, K., see Zhan, H. F.

Yang, Y., see Ren, G.

Ye, H.-F., see Zhang, Z.-Q.

Zhan, H. F., Xia, K. \& Gu, Y. T., Tensile Properties of Graphene-Nanotube Hybrid Structures: A Molecular Dynamics Study
2 (2013) 1350011

3 \& 4 (2013) 1350018

1 (2013) 1350001

2 (2013) 1350012

1 (2013) 1350004

1 (2013) 1350005

$2(2013) 1350010$

2 (2013) 1350008

3 \& 4 (2013) 1350016

1 (2013) 1350001

3 \& 4 (2013) 1350016

1 (2013) 1350004

3 \& 4 (2013) 1350019

1 (2013) 1350004

1 (2013) 1350005

3 \& 4 (2013) 1350018

1 (2013) 1350004

2 (2013) 1350010

2 (2013) 1350012

3 \& 4 (2013) 1350014

3 \& 4 (2013) 1350020

2 (2013) 1350010

3 \& 4 (2013) 1350017

3 \& 4 (2013) 1350020 
Zhang, Y.-W., see Li, K.

Zhang, Z.-Q., Ye, H.-F., Zheng, Y.-G., Cheng, G.-G.,

Ding, J.-N. \& Ling, Z.-Y., Loading, Charging and

Thermal Effects on the Mechanism of Water-Carbon

Nanotube Transmission

Zheng, Y.-G., see Zhang, Z.-Q.
$2(2013) 1350011$

3 \& 4 (2013) 1350017

3 \& 4 (2013) 1350017 